Gotlind Ulshöfer

\title{
Smart Home im Alter als Herausforderung für Diakonie und Diakoniewissenschaft
}

\section{Einleitung: Smart Home und Diakoniewissenschaften}

Das „Smart Home“, das heißt die Vernetzung von Haushaltsgeräten, Unterhaltungselektronik und Haustechnik mit externen Anbietern im eigenen Zuhause, schreitet immer weiter voran. Dabei ist das Thema "Smart Home“ auch für die Diakoniewissenschaften interessant: Diakonische Dienstleistungen finden nicht nur in Heimen und Institutionen statt, sondern auch ambulant, d. h. im Zuhause der Zu-Pflegenden und ihrer Angehörigen und damit auch in privaten Räumen. Blickt man auf die Daten des Statistischen Bundesamts, welche die Diakonie Deutschland auf ihrer Webseite veröffentlich hat, so waren schon 2015 mehr als zwei Drittel aller Pflegebedürftigen zuhause versorgt worden - und damit stieg auch die Nachfrage nach Leistungen der ambulanten Pflegedienste (Diakonie Deutschland 2019).

Dabei wird durch Smart Home der private Raum in doppelter Hinsicht verändert. Erstens gibt es durch Digitalisierung mehr technische Unterstützungsmöglichkeiten für ein selbstbestimmtes Leben im Alter. Zweitens wird der private Bereich aber auch stärker vernetzt und eingebunden in einen Datentransfer, der Daten über die Lebensgewohnheiten der jeweiligen Personen nach außen bringt, wodurch es zu einem erhöhten Monitoring kommt. Dies kann - auf den ersten Blick - gerade für ältere Personen, die zuhause leben und die im Laufe der Zeit immer mehr auf pflegerische Unterstützung angewiesen sind, und für die ambulante Hilfe eine gute Ergänzung zu den bestehenden Angeboten sein. Aber andererseits kann es auch zu vermehrter ungewollter Weitergabe von privaten Daten, die beispielsweise zu Überwachung führen, kommen. So stellen sich in diesem Zusammenhang auch grundlegende diakoniewissenschaftliche Fragen: was bedeuten diese Entwicklungen für ein diakonisches Selbstverständnis? Wird jetzt Pflege immer weiter technisiert und Alter immer weiter normiert? Im Folgenden will ich aus der Fülle der Fragestellungen, die sich aus der Digitalisierung ergeben, diese zuspitzen auf die Frage: Inwiefern ist Diakoniewissenschaft angesichts der Entwicklungen des Smart Home gerade auch für Ältere herausgefordert? Meine These dazu lautet: Die Herausfor- 
derungen für die Diakoniewissenschaft liegen nicht nur bei den ethischen Problemen hinsichtlich der Anwendung der digitalen Möglichkeiten im Smart Home bei Älteren, sondern die Diakoniewissenschaft hat darüber hinaus die Aufgabe, die Prozesse der Digitalisierung als gesellschaftliche Veränderungen zu analysieren und kritisch zu begleiten. Denn die Herausforderungen, die sich im diakonischen Bereich zeigen, finden sich auch gesamtgesellschaftlich wieder.

\section{Smart Home und Leben im Alter}

Zur Illustration, was Smart Home und Leben im Alter bedeuten kann, möchte ich auf den Film „Robot \& Frank - Zwei diebische Komplizen“1 zurückgreifen und dabei insbesondere zwei Aspekte in den Vordergrund stellen: In dem Film „Robot \& Frank“, der in der nahen Zukunft spielt, geht es um einen alten Herrn namens Frank, der von seinem Sohn einen Pflegeroboter, Robot genannt, bekommt. Frank ist ein älterer Herr und Hauptprotagonist, der früher Einbrecher war und jetzt aber ein bürgerliches Leben, allein in einem Haus am Rande einer kleinen US-amerikanischen Stadt lebt - und Robot ist ein auf zwei Beinen, aufrechtstehender und agierender Pflegeroboter, den Franks Sohn ihm gebracht hat. Frank zeigt Anzeichen einer beginnenden Demenz. Der Roboter soll ihm bei der Bewältigung des Alltags helfen, was zunächst von dem alten Frank abgelehnt wird. Der Film ist interessant, weil er uns auf verschiedenen Ebenen für das Thema „Smart Home“ auf wichtige Fragen hinweist:

Erstens ist interessant, dass das „Smart Home“ des älteren Herrn gar nicht so richtig smart ist: zwar gibt es einen Bildschirm, auf dem mit den Kindern telefoniert wird, aber ansonsten ist die größte technische Innovation bei Frank tatsächlich der Roboter. Hier ließe sich nun überlegen, ob im Film diese Zuspitzung aus dramaturgischen Gründen geschah, oder ob sich darin nicht tatsächlich eine treffende Überlegung widerspiegelt: Kann denn gerade bei älteren Menschen von einem digital vernetzten Haus bzw. Wohnung ausgegangen werden? Sind denn bei jedem und jeder überhaupt die finanziellen Ressourcen und der Wille vorhanden, sich technologisch in dem Maße zu vernetzen? Blickt man auf die technologischen Möglichkeiten des Smart Home gerade für Ältere, so fällt die Bilanz, was alles an technischen Hilfsmitteln tatsächlich eingesetzt wird, doch relativ mager aus. Dabei wird in Deutschland das Thema der technisierten Pflege vor allen

1 Robot \& Frank - Zwei diebische Komplizen, engl.: Robot\&Frank, Regie: Jake Schreier, USA 2012. 
Dingen unter dem Stichwort „Ambient Assisted Living“ diskutiert. Es geht hierbei um assistive Systeme, die

„reichen von einfachen Schaltern für Licht, Heizung ... über Sensoren und Kameras zur Überwachung der häuslichen Aktivitäten von älteren und pflegebedürftigen Menschen ... < und um > ... Komplexere Assistenzsysteme, $<$ die $>$... Vitalparameter überwachen und sogar Vitalfunktionen steuern. Nicht zuletzt wird an Servicerobotern zur Substitution von Pflegekräften ... und technischen Unterstützungen zur Kommunikation sowie Substituten für soziale, zwischenmenschliche Kontakte (zum Beispiel die elektronische Robbe Paro) gearbeitet." (Manzeschke 2011, 106)

Dabei sind jedoch nicht viele dieser technischen Möglichkeiten in Privathaushalten im Einsatz (Zhang / Li / Wu 2020, 2). Private Pflegeroboter, wie sie im dem Film Robot \& Frank vorgestellt werden, sind bis jetzt in Deutschland im Vergleich mit beispielsweise Saugrobotern nicht verbreitet. Andererseits wird die Forschung zu AAL oder auch „Smart Home for Seniors" von Seiten der verschiedenen Regierungen und Unternehmen gefördert (Do et al., 2017).

Zweitens zeigt der Film auch, wie aktivierend ein Pflegeroboter sein kann: Frank, der früher Einbrüche gemacht hat, freundet sich langsam mit Robot an. Als er erkennt, dass Robot ihm beim Einbruch und dem Diebstahl von Schmuck bei den arroganten und reichen Nachbarn helfen kann, lernt er ihn zu schätzen. Und Robot lernt relativ schnell, was Frank ihm für die Raubtaten beibringt, wobei ein ethisch-moralisches Gewissen bei ihm nicht einprogrammiert ist. So klappt der Einbruch, aber die Polizei kommt den beiden auf die Spur und so trennen sich die Wege: Robot wird entsorgt bzw. bekommt eine neue Festplatte und Frank kommt ins Altersheim, wo man ihn am Ende des Films einsam, traurig und apathisch in seinem Zimmer sitzen sieht. Zumindest hier im Film hat der Roboter tatsächlich die Autonomie des alleinlebenden alten Menschen gestärkt und seine Lebensqualität verbessert, obwohl der Robot andererseits an einer Straftat beteiligt wurde.

\section{Diakonie in der Digitalisierung: Kennzeichen diakonischen Handelns im Kontext der Arbeit mit Älteren}

Zunächst gilt auch für die Diakonie in der Digitalisierung der biblische Grundsatz aus dem Matthäusevangelium: „Was ihr meinen geringsten Geschwistern getan habt, das habt ihr mir getan“ (Mt 25,40). Er bringt zum Ausdruck, dass diakonisches Handeln im Christusgeschehen verankert ist. Der Satz kann als „fundamentale christliche Handlungsmaxime“ für das 
„Engagement zugunsten von armen und sozial benachteiligten Menschen“ gelten (beide Zitate: Eurich 2019, 31). Heutzutage ist dabei zu berücksichtigen, dass Diakonie sowohl in leiblicher Präsenz als auch online stattfindet - und dazuhin „onlife“ (Floridi 2014), d. h. in dem Konglomerat von online und offline.

So kann die „Diakonie Deutschland“ in dem Entwurf ihrer „Digitalen Agenda“ auch schreiben: „Digitalisierung beschreibt alle Maßnahmen für Hilfesuchende, Angehörige und Mitarbeiter*innen, die durch digitale Kommunikation, digitale Produkte und Prozesse sowie Organisation der ITArchitektur umgesetzt und angeboten werden können. Sie betrifft daher unsere Angebote an die Menschen, aber auch unsere interne Organisation." (Diakonie Deutschland, o. D.) Somit ist auch die ambulante Altenpflege betroffen. Dabei ist das diakonische Angebot im Bereich der Altenpflege reichhaltig und reicht von aktueller Hilfe in Notlagen über die Ermöglichung von mobilem und selbständigem Leben sowie seelsorgerlicher und kultureller Arbeit (Hartmann/Hildemann 2005, 459). All die beschriebenen Aktivitäten der Fürsorge können sowohl von technischen Assistenzsystemen unterstützt oder auch, was die Kommunikationsangebote angeht, mit Hilfe von Informations- und Kommunikationstechnologien vollzogen werden.

Inhaltliche Kennzeichen diakonischen Handeln stellen, wie beschrieben, das Engagement für die Bedürftigen ins Zentrum. Dieses Engagement ist getragen von der Vorstellung der Würde jedes Menschen, worin sich auch Nächstenliebe manifestiert, die sich gleichzeitig in der (Für)-Sorge bzw. care zeigt, und Inklusion und Teilhabe mit sich bringt - so auch der Diakoniewissenschaftlicher Thorsten Moos über die religiöse Rationalität diakonischen Helfens (Moos 2019, 106 ff.). Diese Kennzeichen - Würde, Sorge und Inklusion - sollen für die hier vorliegenden Analysen auch auf ein christlich fundiertes Verständnis von diakonischem Handeln bei alten Menschen in der Digitalisierung bezogen werden: Die Menschenwürde als grundlegender Ausgangspunkt für das diakonische Arbeiten, die sich christlich auch in der Gottebenbildlichkeit des Menschen gründet, führt zur Annahme, dass jeder Mensch aufgrund seines bzw. ihres Menschseins würdig zu behandeln ist. Dies bedeutet gerade für Betagte, dass sie hier in ihrer Individualität und Originalität wahrgenommen und respektiert werden sollten. Es geht also auch um eine Pluralisierung von Altersbildern. Zum anderen kann das Phänomen der Fürsorge, der Care, und damit der Zuwendung zum Nächsten noch weitere Formen annehmen, die sich nicht nur in direkter Begegnung ausdrücken, sondern auch digital vermittelt sein können. Dabei ist zu beachten: „Vielmehr ist hier hervorzuheben, dass im 
Alter das individuelle Bemühen um Aufrechterhaltung der selbständigen Lebensführung zunehmend an Aktualität gewinnt." (Kruse 2005, 275). Dazuhin ergibt sich für die Fürsorge in Würde, dass es auch um Inklusion geht. Diese kann im Sinne der Interpretation des Theologen Wolfhard Schweiker definiert werden, der Inklusion als ein Prinzip versteht ..., „das die Relationalität der sozialen Einbezogenheit des Menschen auf der Grundlage der wechselseitigen Achtung der qualitativ bestimmten Menschenwürde und auf der Grundlage eines nicht ausgrenzenden Menschen- und Wirklichkeitsverständnisses beschreibt und zu sichern beansprucht" (Schweiker 2017, 431, H. i. O.) - und gleichzeitig mit Partizipation, Teilhabe und Freiheit verknüpft ist.

\section{Smart Home, Digitalisierung und das Alter - Herausforderungen für Diakonie und Diakoniewissenschaft}

\subsection{Smart Home und die Würde im Alter}

Das Heim eines Menschen ändert sich, wenn es zum Smart Home wird, denn die Trennlinie zwischen „außen“ und „innen“, zwischen „privat" und „öffentlich“ ist nicht mehr so klar zu ziehen - und dies insbesondere nicht, wenn es auch um den Datenaustausch geht. Dabei stellt sich auch die Frage, was dies für die Würde von Älteren angesichts der Nutzung digitaler Möglichkeiten bedeutet:

Erstens geht es darum, die Pluralität von Formen des Alt-Seins, von Altersbildern, zu ermöglichen und auszuweiten. Dazu können Narrative, wie beispielsweise Filme - wie Robot \& Frank - oder auch Geschichten dienen. So können wir zu Phantasien gelangen, wie unsere zukünftigen Lebenswelten aussehen könnten - und können darüber diskutieren, ob dies eine Variante ist, wie wir die technischen Geräte nutzen wollen bzw. wie diese aussehen sollten. Der Film Robot \& Frank stellt auch einen Beitrag dar, Bilder von alten Menschen zu pluralisieren und in einem positiven Licht zu betrachten, denn trotz der beginnenden Demenz hat Frank Spaß am Leben und arbeitet mit Robot an einem Ziel. Dabei sind die Bilder vom Alter vielfältig, insbesondere, wenn man sie aus einer interkulturellen Perspektive betrachtet (Hock 2014). Diese Vielfalt, in den Formen alt zu sein, entspricht der Würde jedes Menschen, die gerade unter christlichen Gesichtspunkten betrachtet in jedem Menschen Gottes Ebenbild sieht, egal wie alt jemand ist. Technische Hilfsmittel können in diesem Kontext tatsächlich zur Aufrechterhaltung von Selbständigkeit führen, trotz zuneh- 
mender Verletzlichkeit und Abhängigkeit im Alter, und damit die Würdigkeit des Alters gestalten helfen.

Zweitens stellen sich aber gerade angesichts den Vernetzungsmöglichkeiten, die das Smart Home bietet, zum einen die Fragen, wie die Technikaffinität gerade von älteren Personen zu beurteilen ist. Wie Nutzer*innenfreundlich sind die entsprechenden Geräte? Selbst hinsichtlich des schon länger eingeführten Hausnotrufs zeigen Untersuchungen Defizite im Hinblick auf das Nutzungsverhalten, das die Forschenden auch der Art der Geräte zuschreiben und schlussfolgern: „Daraus wird deutlich, dass der reflektierte Umgang mit der Technologie selbst wenig entwickelt ist, obwohl sie schon seit langem fast unverändert im Einsatz ist." (Klein/ Reutzel/ Roßberg 2015, 166). Zum anderen stellt sich aber auch die grundlegende Frage, inwiefern bei einem kompletten Smart Home, das also sowohl Roboter wie Robot umfasst, als auch mit „smarter Kleidung“ die Körperfunktionen der Bewohner*innen vermisst und mit Kameras und sensiblen Böden die Bewegungsprotokolle der Bewohner*innen erstellt, die Lebensführung der älteren Personen komplett überwacht wird und wie sich dies dann auf das Selbstverständnis und Verhalten der Bewohner*innen auswirkt. Damit einher geht dann auch die Frage, nach welchen Kriterien die Daten ausgewertet werden - gibt es beispielsweise ein Spektrum, in dem Bewegungsabläufe der älteren Personen normiert werden, damit die jeweiligen Sensoren entsprechend Alarm ausschlagen, wenn dieses Limit über- oder unterboten wird? Was bedeutet dies dann für die Betroffenen? Weiter zugespitzt formuliert: Ist die Aufgabe der Freiheit der Preis der Überwachung? Diese Frage kann an die Diskussionen im vergangenen Jahr bezüglich der Corona-App und auch an den Umgang und die CoronaMaßnahmen in anderen Ländern wie China, die keinen großen Wert auf Datenschutz legen, erinnern. Daran zeigt sich, dass die Diskussionen, die angesichts der potentiellen Möglichkeiten des Smart Homes für Ältere auch hinsichtlich der Entwicklungen einer fairen Gesellschaft zu führen sind. Denn: Es geht gerade aus einer diakonischen Perspektive insbesondere darum, die „Option für die Armen und Schwachen“ bei den Diskussionen im Blick zu behalten und dabei immer wieder daran zu erinnern, nicht in einem paternalistischen Sinne für die Verletzlichen sprechen zu wollen, sondern mit ihnen und wenn der Bedarf bei den Verletzlichen vorhanden ist, bei Bedarf auch für sie. Die Diskussionen um Smart Home und Alter spiegeln also grundlegende Diskussionen wider. Sie zeigen mit Hilfe der Analysen zum Einsatz und zur Nutzung von Technik bei Älteren, wie wichtig es ist, „dass Technik gestaltbar ist und an den Menschen angepasst werden muss" (Hurtienne 2016, 267). 


\subsection{Digitalisierung und Care - die Pflegenden im Blick}

Herausfordernd für die Diakonie ist angesichts des Smart Home auch, inwiefern sich die Formen der Zuwendung in der Pflege durch die technologischen Möglichkeiten wandeln. Verändert das Smart Home auch die Bedeutung häuslicher Pflege? Wie müssen technische Möglichkeiten gestaltet sein, damit technisierte Pflege als eine soziale und interaktive Begegnung zwischen Menschen erhalten bleibt? Wenn man sich die Diskussionen um „Smart Home for Seniors" oder auch die Werbung für technische Assistenzsysteme anschaut, so finden sich dort die Versprechen für eine Erleichterung in der Pflege:

„Wenn Lastenroboter Transport- und Botendienste übernehmen, Ortungssysteme von Überwachungs- und Kontrollaufgaben entlasten und sensorgestützte Aktivitätsbestimmung das Monitoring bei gesundheitlich vulnerablen Menschen ermöglicht, sollen damit Möglichkeiten zur Konzentration auf personengebundene Aufgaben in der Pflege sowie zur Kompensation des drohenden Fachkräftemangels aufgezeigt werden." (Hülsken-Giesler 2015, 117)

In einer explorativen Studie zur Nutzung technischer Assistenzsysteme wie Sensoren kommt Hülsken-Giesler zu dem Ergebnis, dass Sensoren zwar weitere Informationen über die ältere Person zuhause liefern, dass es aber erst der direkte Kontakt und Austausch ist, der zu einer besseren und angemesseneren Einschätzung der Situation führt: „Diese Form der kontextgebundenen, häufig durch alltagsweltlich orientierte Kommunikationen begleitete Situationseinschätzung vor Ort lässt sich, den Pflegeexperten folgend, kaum durch eine technikgestützte Informationssammlung ersetzen." (Hülsken-Giesler 2015, 122). Obwohl bei der Untersuchung die Datenerhebung der Älteren für die Pflege als sehr positiv eingeschätzt wird, kam die Untersuchung doch auch zur Erkenntnis, dass „Technik zum Störfaktor in der Interaktion zwischen Pflege und Patient werden könnte" (HülskenGiesler 2015, 128). Hierbei zeigt sich die Ambivalenz des Einsatzes der Technologien. Sie sind insofern als Herausforderung für die Diakonie bzw. auch die Diakoniewissenschaften zu verstehen, weil für die Entwicklung, Nutzung und den Einsatz der Technologien im Rahmen von Smart Home genaue Abwägungsprozesse entwickelt werden müssen, wie es zu einem diakonisch verantwortbaren Einsatz kommen kann. 


\subsection{Organisationsformen angesichts der Digitalisierung und die Frage nach der Inklusion und Teilhabe}

Die Möglichkeiten, die Smart Home gerade auch hinsichtlich der Pflege im Alter bietet, haben mindestens zwei organisatorische Auswirkungen, die für die Diakonie und damit auch für die Diakoniewissenschaft in Zukunft verstärkt bedacht werden müssen. Zum einen - und dies wird seitens der Diakonischen Werke insbesondere auch aus rechtlicher und ökonomischer Sicht schon in Betracht gezogen -, geht es um die Organisation der Hilfeleistungen über Plattformen und Apps (zur rechtlichen Lage vgl. die Informationen des Diakonischen Werks: https://www.diakonie.de/journal/rech tsrahmen-digitalisierung) und auch hier gilt es die Chancen und Grenzen nicht nur unter rechtlichen Gesichtspunkten, sondern dazuhin aus einer diakoniewissenschaftlichen Perspektive zu diskutieren und die Frage nach den Teilhabemöglichkeiten und Exklusionsmechanismen zu stellen, in ihrer ganzen Ambivalenz. Zum anderen gilt es zu durchdenken, was die „Technologies of Care", also Sorgetechnologien, wie Stephen Webb und Elizabeth Harlow (Webb/Harlow 2003) sie nennen, mit den Pflegenden machen bzw. wie sich die digitalen Organisationsformen auf die beteiligten Pflegenden auswirken. Zum einen führen Webb und Harlow an, dass die „internetbasierten Informations- und Dokumentationsinstrumente, institutionelle Softwarelösungen, Computerisierung im Arbeitsalltag von Fachkräften ... und der gesamten Steuerung im Gesundheits- und Sozialwesen" (Polutta 2015, 57) und die damit zusammenhängenden Standardisierungsprozesse als positives Element für den pflegerischen Alltag erscheinen. Der Zugriff auf die Daten und Informationen ist standardisierte Teilhabe am Arbeitsprozess. Andererseits kann es aber auch gerade durch die Sozialtechnologien dazu kommen, dass ausgeschlossen wird, was nicht in den digitalen Strukturen erfasst werden kann, wobei es sich um Verhalten oder um Informationen handeln kann. Dies kann dann wiederum zu Ausschlussmechanismen führen oder zu unangebrachten Normierungen.

Außerdem stellt sich hinsichtlich der Bereitstellung technischer Geräte im Smart Home für die Älteren nicht nur die Frage nach den Nutzungsmöglichkeiten, sondern auch, wer dafür kostenmäßig aufkommen sollte. Für Ältere kann die Nutzung technischer Gerätschaften - wenn sie mit entsprechenden Abrechnungsmöglichkeiten verankert ist und damit für alle zugänglich gemacht wird -, tatsächlich zu einer Möglichkeit zur stärkeren Inklusion ins gesellschaftliche Leben führen, was ihnen sonst nicht mehr möglich wäre, weil beispielsweise die eigene Wohnung nicht mehr verlassen 
werden kann. Aber wenn diese Möglichkeiten nicht für alle, die sie benötigen, finanziert werden können, kommt es wiederum zur Exklusion. Als Herausforderung für die Diakonie stellt sich daher die Beurteilung dar, wann die „Technologies of Care“ zu welchem Ergebnis führen und welche Voraussetzungen es für eine Bereitstellung und Nutzung im Sinne von Teilhabe, Würde und Care braucht.

\section{Diakoniewissenschaft als „Digital Diaconic Studies“}

Angesichts der genannten Herausforderungen gilt es die Diakoniewissenschaften zu öffnen. Ulrich H. J. Körtner definiert Diakoniewissenschaft wie folgt:

„Diakoniewissenschaft ist ein praxisorientierter Verbund von unterschiedlichen Wissenschaftsdisziplinen aus Theologie, Humanwissen- und Sozialwissenschaften sowie der Wirtschaftswissenschaft (Ökonomik). Ihre innere Einheit wird durch ihren Gegenstand, nämlich das diakonische Handeln, geschaffen, dessen Begriff theologisch zu bestimmen ist." (Körtner 2017, 2)

Über die Definition hinaus, die mit dem Praxisbezug und der Interdisziplinarität wesentliche Merkmale nennt, geht es darum, die Diakoniewissenschaft zu „digital diaconic studies“ weiterzuentwickeln. Angesichts der digitalen Entwicklungen muss es darum gehen, einerseits die Erkenntnisse diakoniewissenschaftlicher Forschung aufzunehmen und weiter zu tradieren und gleichzeitig die Diakoniewissenschaften für digitale Zusammenhänge zu öffnen - und zwar sowohl inhaltlich als auch methodologisch. Zur Illustration greife ich auf die Analysen der US-amerikanischen Theologin und Religionswissenschaftlerin Heidi Campbell und des in Neuseeland lehrenden Stephen Garner zurück. Die beiden Wissenschaftler*innen haben in ihrem Werk „Networked theology“ (Campbell/Garner 2016) herausgearbeitet, dass Theologie angesichts der Digitalisierung zum einen Netzwerkstrukturen bedenken sollte und in diesem Sinne die Kontextualität jedes Theologietreibens berücksichtigen sollte und zum anderen - ausgehend von der Netzwerkperspektive - auch digitale Zusammenhänge wahrnehmen sollte. Wird dieser Ansatz auf die Diakoniewissenschaften bezogen, so ergibt sich ein dezidierter Praxisbezug und eine interdisziplinäre Ausrichtung, im Sinne einer „kombinatorischen Wissenschaft“ (Geyer 2015, 146) - und es geht auch darum, die Möglichkeiten digitaler Vernetzung mit seinen Chancen und Grenzen zu untersuchen und somit auch gesellschaftlich einen Beitrag zur Entwicklung, wie Digitalisierung eingesetzt werden kann 
und soll, zu leisten. Dabei sind drei Ebenen der Analyse zu unterscheiden (Eurich 2019, $37 \mathrm{f}$ ):

Erstens gilt es die Handlungsebenen vor Ort in den Blick zu nehmen, also zu analysieren, z. B. was und wie mit Hilfe „smarter Technologien“ den Alltag von Unterstützungsbedürftigen und Pflegenden sowie Angehörigen ermöglicht, erleichtert oder auch einschränkt. Hierbei kann dann ein ethisches Evaluationsmodell, wie Arne Manzeschke und Kolleg*innen mit MEESTAR vorgelegt haben, genutzt werden. Das Modell dient „zur ethischen Evaluation sozio-technischer Arrangements“, in dem der Einsatz von neuen Technologien unter den Aspekten von Fürsorge, Selbstbestimmung, Sicherheit sowie auf den verschiedenen Ebenen des Individuellen, Organisatorischen und Gesellschaftlichen ethisch beurteilt wird (Manzeschke et al. 2013).

Zweitens geht es um die Analysen zum christlichen Deutungshorizont des diakonischen Handelns im Rahmen von digitalen Möglichkeiten. Wie lässt sich mit Hilfe der Technik bzw. sogar in der Technik selbst - sei es in ihrem Design oder in ihrem Einsatz - christliche Orientierung im Sinne von Würde, Care und Inklusion, beim diakonischen Handeln einbringen? Der „Deutungshorizont“ bedeutet, dass diakonisches Handeln aus einer grundlegenden Einsicht geschieht, dass Menschen Geschöpfe Gottes sind und dass insbesondere der Einsatz für arme und marginalisierte Menschen aus einer christlichen Perspektive zentral ist. Meines Erachtens sind die theologischen Überlegungen, wie ein christlich geprägter Deutungshorizont gerade auch in einer digital vernetzten Welt zum Tragen kommen kann, wichtig, denn hieraus lassen sich Beiträge für einen gesellschaftlichen Diskurs um ethische Perspektiven für den Einsatz der Technologien entwickeln.

Drittens geht es um „kontextuelle Aspekte, die die Struktur und den Rahmen diakonischen Handelns betreffen“ (Eurich 2019, 38) - und hierbei zeigt sich, dass es bei den Diakoniewissenschaften nicht nur um ethische Fragen geht, sondern auch darum, organisatorische Strukturen und (sozial-)politische Gestaltung zu durchdenken und zu analysieren - und dies ist gerade auch in der Situation wichtig, in der wir uns im Augenblick hinsichtlich der Entwicklungen des "Smart Homes“ befinden, denn hier ist noch relativ viel offen und damit erst „im Werden“, was es gesellschaftlich, ökonomisch, rechtlich, informationswissenschaftlich, architektonisch, politisch und eben auch unter diakonischen Gesichtspunkten zu diskutieren und zu entscheiden gilt: Dazu können Diakonie und Diakoniewissenschaft einen Beitrag leisten. 


\section{Literaturverzeichnis:}

Campbell, Heidi A. und Stephen Garner. 2016. Networked Theology. Negotiating Faith in Digital Culture. Grand Rapids: Baker Academic.

Diakonie. 2019. „Pflegestatistik 2015.“ https://www.diakonie.de/statistik/1 12018-pflegestatistik-2015 [Zugriff: 30.12.2020].

Diakonie. O. D. „Digitale Agenda.“. https://www.diakonie.de/digitale-agen da/ [Zugriff: 30.12.2020]

Do, Ha Manh, Minh Pham, Weihua Sheng, Dan Yang und Meiquin Liu. 2017. "RiSH: A Robot-integrated Smart Home for Elderly Care." Robotics and Autonomous Systems Volume 101 (March): S. 74-92. https://doi.o rg/10.1016/j.robot.2017.12.008 [Zugriff: 30.12.2020].

Eurich, Johannes. 2019. „Kritisch-konstruktive Auseinandersetzung mit dem diakonischen Profil." In Diakoniewissenschaften in Forschung und Lehre 2018/2019 Bd 46, Hrsg. Johannes Eurich, Dorothea Schweizer, S. 29-38. Heidelberg: DWI Jahrbuch. https://doi.org/10.11588/dwijb.2 019.46

Floridi, Luciano. 2014. The Fourth Revolution. How the Infosphere is Reshaping Human Reality, Oxford: Oxford University Press.

Geyer, Christian. 2015. „Diakoniewissenschaft - Theologie - Diakoniemanagement. Versuch einer Ortsbestimmung in Thesen." In Diakoniewissenschaft und Diakoniemanagement an der Kirchlichen Hochschule Wuppertal/Bethel. Interdisziplinarität, Normativität, Theorie-Praxis-Verbindung, Hrsg. Matthias Benad, Martin Büscher, Udo Krolzik, S. 145-150. Baden-Baden: Nomos.

Hartmann, Klaus und Klaus Hildemann. 2005. „Altenhilfe.“ In Diakonisches Kompendium, Hrsg. Günter Ruddat, Gerhard K. Schäfer, S. 455466. Göttingen: Vandenhoeck \& Ruprecht.

Hock, Klaus. 2014. „Grenzenlos altern? Transkulturelle Altersbilder zwischen religiöser Rückbindung und säkularem Imperativ." In Lebensqualität im Alter. Gerontologische und ethische Perspektiven auf Alter und Demenz, Hrsg. Michael Coors, Martina Kumlehn, S. 37-56. Stuttgart: Kohlhammer.

Hülsken-Giesler, Manfred. 2015. „Technische Assistenzsysteme in der Pflege in pragmatischer Perspektive der Pflegewissenschaft. Ergebnisse empirischer Erhebungen." In Technisierung des Alltags. Beitrag für ein gutes Leben?, Hrsg. Karsten Weber, Debora Frommeld, Arne Manzeschke, Heiner Fangerau, S. 117-130. Stuttgart: Steiner. 
Hurtienne, Jörg. 2016. „Techniknutzung im Alter.“ In Alter und Altern. Herausforderungen für die theologische Ethik, Hrsg. Stephan Ernst, S. 251270. Fribourg: Herder Verlag.

Klein, Barbara, Sebastian Reutzel und Holger Roßberg. 2015. „Zur Mediatisierung assistiver Technologien - der Hausnotruf als Kommunikationsmedium für ältere Menschen." In Mediatisierung (in) der Sozialen Arbeit, Hrsg. Nadia Kutscher, Thomas Ley, Udo Seelmeyer, S. 151-168. Baltmannsweiler: Schneider-Verlag Hohengehren.

Körtner, Ulrich H. J. 2017. Diakonie und Öffentliche Theologie. Diakoniewissenschaftliche Studien. Göttingen: Vandenhoeck \& Ruprecht.

Kruse, Andreas. 2005. „Selbständigkeit, bewusst angenommene Abhängigkeit, Selbstverantwortung und Mitverantwortung als zentrale Kategorien einer ethischen Betrachtung des Alters." Zeitschrift für Gerontologie und Geriatrie 38 (4): S. 273-287.

Manzeschke, Arne. 2011. „Tragen technische Assistenzen und Robotik zur Dehumanisierung der gesundheitlichen Versorgung bei? Ethische Skizzen für eine anstehende Forschung. "In Technisierte Medizin - Dehumanisierte Medizin? Ethische, rechtliche und soziale Aspekte neuer Medizintechnologien, Hrsg. Kirsten Brukamp, Katsiaryna Laryionara, Christoph Schweikhardt, S. 105-112. Kassel: Kassel University Press.

Manzeschke, Arne, Karsten Weber, Elisabeth Rother und Heiner Fangerau. 2013. Ergebnis der Studie „Ethische Fragen im Bereich Altersgerechter Assistenzsysteme“. Ludwigsfelde: VDI/VDE Innovation + Technik.

Moos, Thorsten. 2019. „Religiöse Rationalität des Helfens. Systematischtheologische Beiträge zu einer Theorie diakonischer Praxis." Zeitschrift für Evangelische Ethik 63 (2): S. 104-116.

Polutta, Andreas. 2015. „Technologies of Care und wirkungsorientierte Steuerung. $\mathrm{Zu}$ aktuellen Transformationsprozessen in der Sozialen Arbeit." In Mediatisierung (in) der Sozialen Arbeit, Hrsg. Nadia Kutscher, Thomas Ley, Udo Seelmeyer, S. 56-76.. Baltmannsweiler: SchneiderVerlag Hohengehren.

Schweiker, Wolfhart. 2017. Prinzip Inklusion. Grundlagen einer interdisziplinären Metatheorie in religionspädagogischer Absicht. Göttingen: Vandenhoeck \& Ruprecht.

Webb, Stephen A. und Elizabeth Harlow. 2003. Information and Communication Technologies in the Welfare Services. London: Jessica Kingsley Publishers. 
Zhang, Quan, Meiyu Li, Yijin Wu. 2020. "Smart Home for Elderly Care: Development and Challenges in China." BMC Geriatrics 20:318https://d oi.org/10.1186/s12877-020-01737-y [Zugriff: 30.12.2020]. 
